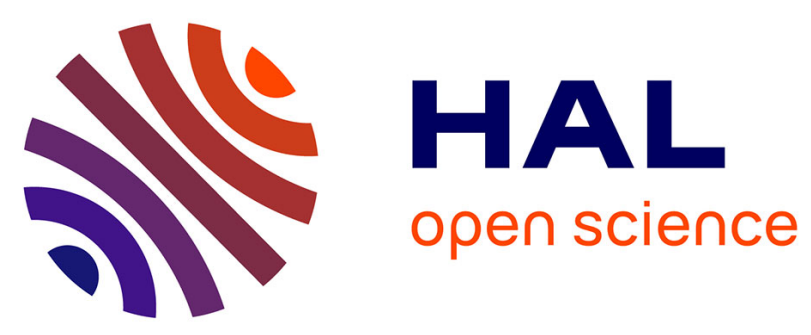

\title{
Multi-modal vortex- and rain-wind- induced vibrations of an inclined flexible cable
}

\author{
Donglai Gao, Wen-Li Chen, Run-Tao Zhang, Ye-Wei Huang, Hui Li
}

\section{To cite this version:}

Donglai Gao, Wen-Li Chen, Run-Tao Zhang, Ye-Wei Huang, Hui Li. Multi-modal vortex- and rainwind- induced vibrations of an inclined flexible cable. Mechanical Systems and Signal Processing, 2019, 118, pp.245-258. 10.1016/j.ymssp.2018.08.057 . hal-02563614

\section{HAL Id: hal-02563614 https://hal.science/hal-02563614}

Submitted on 11 May 2020

HAL is a multi-disciplinary open access archive for the deposit and dissemination of scientific research documents, whether they are published or not. The documents may come from teaching and research institutions in France or abroad, or from public or private research centers.
L'archive ouverte pluridisciplinaire HAL, est destinée au dépôt et à la diffusion de documents scientifiques de niveau recherche, publiés ou non, émanant des établissements d'enseignement et de recherche français ou étrangers, des laboratoires publics ou privés. 


\title{
Multi-modal vortex- and rain-wind- induced vibrations of an inclined flexible cable
}

Donglai Gao ${ }^{1,2,3}$, Wen-Li Chen ${ }^{1,2, *}$, Run-Tao Zhang ${ }^{1,2}$, Ye-Wei Huang ${ }^{1,2}$, Hui Li ${ }^{1,2}$

${ }^{1}$ Key Lab of Smart Prevention and Mitigation of Civil Engineering Disasters of the Ministry of Industry and Information Technology, Harbin Institute of Technology, Harbin, 150090 China

${ }^{2}$ Key Lab of Structures Dynamic Behavior and Control of the Ministry of Education, Harbin Institute of Technology, Harbin, 150090 China

${ }^{3}$ Aix-Marseille Univ., CNRS, Centrale Marseille, IRPHE, Marseille, 13013 France

\begin{abstract}
We reproduce multi-modal vortex-induced vibrations (VIVs) and multi-modal rainwind-induced vibrations (RWIVs) of an inclined and yawed cable in wind tunnel tests. The flexible cable model has low mass and low damping. First, the cable model is kept dry and exposed to uniform airflow; it experiences first-, second- and third-mode VIVs with the increase of wind speed. The structural responses of VIVs are analysed and the frequency lock-in phenomenon is observed for different modes of VIVs of the flexible cable. In addition to VIVs of the dry cable, RWIVs are excited by guiding water rivulets on the cable surface from a water tube. The first-, second- and third-mode RWIVs of the flexible cable are observed and identified at a much higher range of incoming wind speeds than that of the VIVs. To further explore the origin of the multi-modal behaviours of RWIVs, the upper rivulet is guided and restricted to forming locally along the cable. Experimental results reveal that the higher- and multiple-mode RWIVs can


be excited by a local rivulet, even under a lower wind speed. Finally, RWIVs are compared to VIVs to uncover their underlying similarities and relationships.

Keywords: flexible cable, vortex induced vibrations, rain-wind induced vibrations, multi-modal behaviours

\section{Introduction}

Because of their inherent flexibility, low damping and relatively low mass, long stay cables of cable-stayed bridges are sensitive to wind-induced vibrations. Vortex-induced vibrations (VIVs) and rain-wind-induced vibrations (RWIVs) have been issues of great concern to the bridge engineering community, because the large-amplitude vibrations may lead to fatigue and durability problems. For example, cable-deck connections may suffer from failure, owing to the breakdown of protections against corrosion $[1,2]$.

When subjected to wind, stay cables are characterised by flow separation and alternating vortex shedding into the wake. Periodic vortex shedding can result in large unsteady cross-flow forces acting on the cable [3]. If the shedding frequency approaches an order of the modal frequency of the flexible cable, a cable-wake resonance develops. This fluid-structure synchronization, often referred to as 'lock-in', may induce significant cross-flow vibrations of the cables. In bridge engineering, multimodal VIVs on stay cables are often associated with the non-uniformity of the incoming flow, because bridges are usually constructed in the atmospheric boundary layer [4]. Chen et al. [5] conducted a numerical study, suggesting that a 100-m inclined cable would experience multi-modal VIVs under different velocity profiles. Chen et al. [6] 
experimentally investigated a flexible cable subject to sheared incoming flow. Results revealed that the cable model experienced single- and multi-modal VIVs under different incoming velocity profiles, and the vortex shedding frequencies from the cable synchronised well with the cable's modal frequencies.

Hikami and Shiraishi [7] found that the amplitude of wind-induced vibrations of stay cables could be greatly amplified if they are simultaneously subject to light-to-moderate rain conditions. Owing to great vibration amplitudes, RWIVs have become a major concern for bridge engineers over the past three decades. Vigorous efforts have been made to explore the excitation mechanism of severe cable vibrations under rain and wind conditions. Various research philosophies and approaches, including theoretical modelling [8]-[10], field observation [11]-[13], computational fluid dynamics $[14,15]$ and wind tunnel tests [16]-[23] have been employed to obtain a better understanding of the behaviours and excitation mechanisms of RWIVs.

This type of severe cable vibration, jointly induced by rain and wind, is characterised by much lower frequencies and much larger amplitudes than conventional VIVs. It is generally recognised that RWIVs often occur for wind-speed ranges of 8 to $15 \mathrm{~m} / \mathrm{s}$, in the frequency range of $1-3 \mathrm{~Hz}$ and with yaw angles $20-60^{\circ}$, as concluded by Flamand [16]. Additionally, the upper rivulet on the leeward side of the cable surface and its circumferential oscillation play a crucial role in the excitation of RWIVs. Several wind tunnel tests [16]-[23] have been conducted to gain insights into the excitation mechanism of RWIVs; they considerably improved our understanding of this phenomenon. However, during previous wind tunnel tests, rigid and segmental models 3 
were usually employed because of the limitation of the test sections. Thus far, the multimodal behaviours of RWIVs on a stay cable have never been reproduced or investigated in a wind tunnel test. An alternative approach to studying multi-modal RWIVs is field monitoring. Ni et al. [11] measured the response characteristics and excitation mechanisms of stay cables experiencing RWIVs on the Dongting Lake Bridge, P. R. China. Field monitoring was realised by employing accelerometers, anemometers and rain gauges. The dominant frequency of RWIVs corresponded to the third mode frequency of the cable, and the first five modes were found to participate. Zuo et al. [12] developed a long-term full-scale monitoring system on the Fred Hartman Bridge, United States, to investigate the characteristics of stay-cable vibrations. They attempted to interpret both VIVs and RWIVs in the context of the three-dimensional nature of the environment. By investigating the similarities and differences between VIVs and RWIVs, they argued that RWIVs might be a vortex-induced type of excitation that is different from conventional vortex shedding. This exhibited similarities to the research findings of Matsumoto et al. [24], in which RWIV was explained as a type of VIV at a high reduced velocity. It was shown by experiments that the upper rivulet on the cable surface and the incoming turbulence played crucial roles in the mechanism of the VIV at a high reduced velocity. Zuo and Jones [13] reported a type of large-amplitude vibration of dry cables at a high reduced velocity. They proposed that the largeamplitude cable vibrations, with or without rainfall, might originate from the same type of vortex-shedding mechanism that is different from the von Kármán vortex shedding. Moreover, such a mechanism would be promoted and enhanced by the upper rivulet. 
In the present study, we reproduce multi-modal VIVs and multi-modal RWIVs by employing an inclined flexible cable model in a wind tunnel test. The VIVs are reproduced under a smooth incoming airflow, and the RWIVs are excited under the joint action of a smooth incoming flow and a guided upper rivulet.

This paper is organised as follows. Details of the flexible cable model and the experimental setup are given in Section 2. Experiment results, including multi-modal VIVs and multi-modal RWIVs, excited by either increasing wind speed or local rivulets of the flexible cable, are presented and analysed in Section 3. Conclusions are drawn in Section 4.

\section{Experimental setup}

\subsection{Introduction to the laboratory facilities}

Our experimental study was performed in the larger test section of the Joint Laboratory of Wind Tunnel and Wave Flume (WTWF), Harbin Institute of Technology, P. R. China. The closed-loop wind tunnel laboratory has two test sections, a larger one and a smaller one, as sketched in Fig. 1(a). The dimension of the larger test section is $6.0 \mathrm{~m}$ (width) $\times 3.6 \mathrm{~m}$ (height) with a length of $50 \mathrm{~m}$, whereas that of the smaller one is $4.0 \mathrm{~m}$ (width) $\times 3.0 \mathrm{~m}$ (height) $\times 25 \mathrm{~m}$ (length). A wave flume, as deep as $26 \mathrm{~m}$, lies beneath the larger test section. The wave flume is separated from the larger test section by movable and perforated floors, as noted in Figs. 1(a) and 1(c). The perforated floor allows an immediate water drainage into the wave flume. Therefore, the larger section of the WTWF laboratory was suitable for performing our experimental study of RWIVs. 


\subsection{Flexible cable model and experimental setup}

To excite multi-modal vibrations, the flexible cable model needs to be sufficiently long, within the limitations of the larger test section of WTWF. A cable having a total length, $L$, of $8.31 \mathrm{~m}$ and an outer diameter, $D$, of $98.36 \mathrm{~mm}$ was manufactured to reproduce multi-modal VIVs and RWIVs for our experiment. The flexible cable model realises low mass and low damping ratios on a $12-\mathrm{mm}$ diameter steel wire rope inside with multiple layers of foam taps wrapped around it, as illustrated in Fig. 1(b). These are the key factors influencing the occurrence of VIVs and, particularly, RWIVs. A polyolefin heat-shrink tube was adopted as the outside coating to create a smooth surface for the flexible cable model. The model was tensioned by two rigid support frames, both firmly fixed between the tunnel floor and the ceiling. Thus, the support frames were static, experiencing no detectable vibrations during the wind tunnel tests.

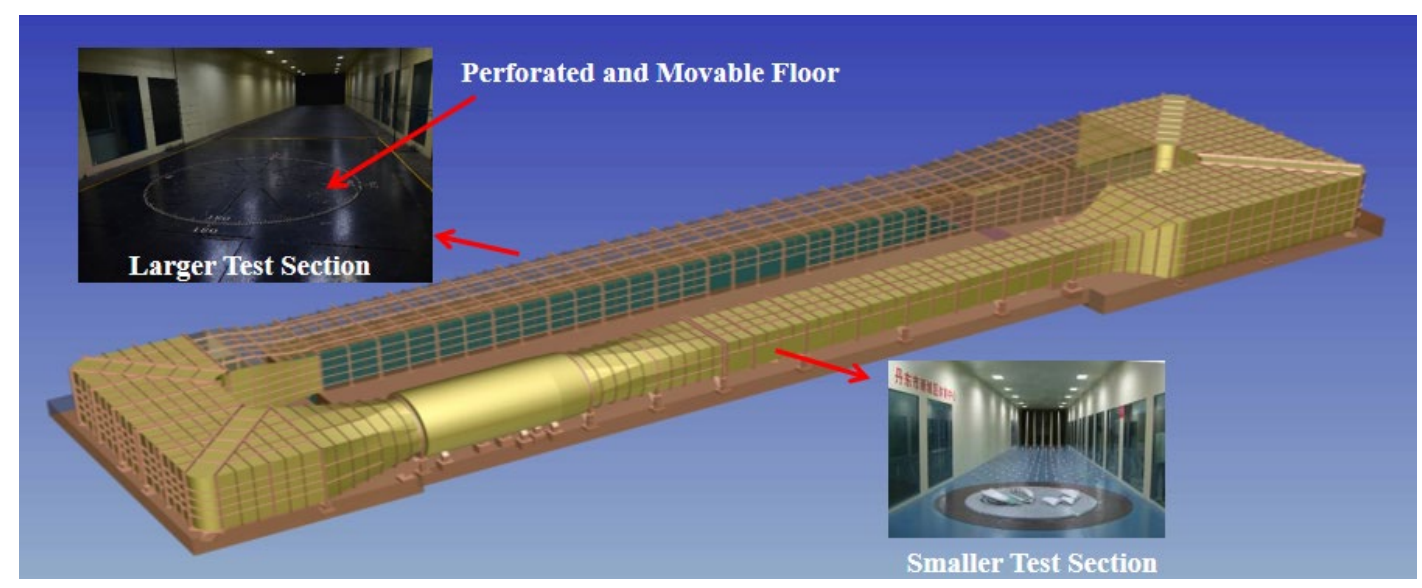

(a) 


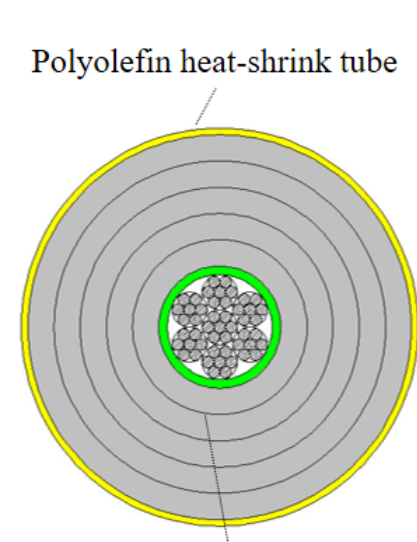

Multi-layer foam tapes

(b)

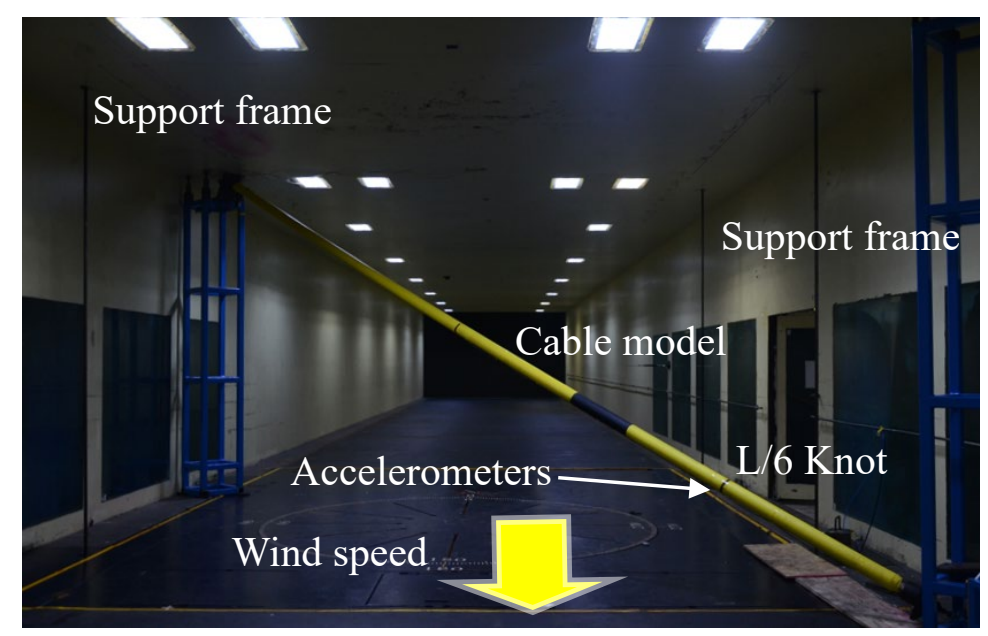

(c)

Figure 1. Cable model and experimental setup: (a) 3D-sketch of the WTWF laboratory; (b) cross-section of the flexible cable model and (c) the inclined flexible cable model.

Two accelerometers (B\&K 4507B, sensitivity: 10.06 and $10.08 \mathrm{mV} / \mathrm{ms}^{-2}$, calibration constant: 98.67 and $98.90 \mathrm{mV} / \mathrm{g}$ ) were fixed at the $L / 6$ knot to measure the in-plane (cross-flow) and out-of-plane (in-line) vibration responses of the flexible cable undergoing VIVs and RWIVs. As shown in Fig. 1(c), the accelerometers were neatly wrapped by water-resistant tapes to avoid the influences of running water. With a sampling frequency of $1000 \mathrm{~Hz}$, the sampling time for each test case was $60 \mathrm{~s}$. Free vibration tests were first conducted in the still air to obtain structural parameters of the flexible cable models. The cable's modal frequencies and damping ratios, defined as the logarithmic damping decrements, were identified based on the free vibrational time time-histories. The results are given in Table 1. Additionally, the mass of the cable model was measured to be $1.03 \mathrm{~kg} / \mathrm{m}$. As shown in Fig. 2(a), the cable's yaw angle, $\beta$, was set as $45^{\circ}$, and its inclination angle, $\alpha$, was decided by the size of the test section to be $23.39^{\circ}$. Both are appropriate conditions for the generation of RWIVs. 
Table 1. Modal frequencies and damping ratios of the flexible cable model.

\begin{tabular}{|c|c|c|c|c|}
\hline & \multicolumn{2}{|c|}{ In-plane } & \multicolumn{2}{c|}{ Out-of-plane } \\
\hline Mode & Frequency (Hz) & Damping ratio (\%) & Frequency (Hz) & Damping ratio (\%) \\
\hline First & 2.31 & 0.41 & 2.14 & 0.39 \\
\hline second & 4.17 & 0.43 & 4.03 & 0.42 \\
\hline Third & 6.36 & - & 6.21 & \multicolumn{2}{c}{} \\
\hline
\end{tabular}

The upper rivulet plays a crucial role in the occurrence of RWIVs. Spraying water in the wind tunnel has been a long-established method of forming an upper rivulet for the reproduction of RWIVs [7, 14]. However, some recent studies [25, 26] successfully excited RWIVs by guiding water lines on the cable surface. It was verified that this approach had the same effects as spraying water. In our study, a plastic water tube with an inner diameter of $5 \mathrm{~mm}$ was glued onto the upper end of the cable model to guide the upper water rivulet and to generate RWIVs, as shown in Fig. 2(b). It is like the configuration made by Jing et al. $[25,26]$, who placed a large water tank outside the wind tunnel, allowing the water flow to be controlled by a valve. In our experiments, the outlet flow rate from the water tube was constantly kept to $21.14 \mathrm{ml} / \mathrm{s}$. This flow rate is relatively large but highly necessary for the reproduction of RWIVs. We found that the upper rivulet could hardly be formed with smaller tube diameters. Thus, the RWIVs could never be reproduced. However, during the RWIVs, only a small amount of water was witnessed to have been blown out by the wind. Even less water contributed to the formation of the upper rivulet. Therefore, most of the guided water are manipulated by gravity to form the lower rivulet. Therefore, the lower rivulet was found to be notably thick, resulting in added mass to the cable model. 


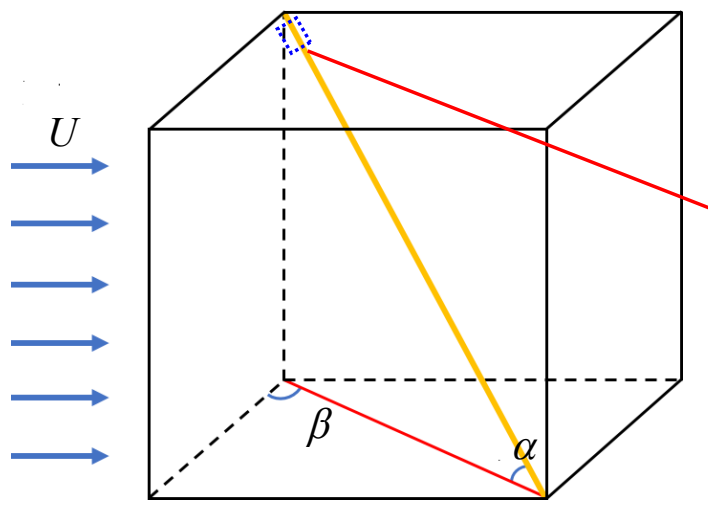

(a)

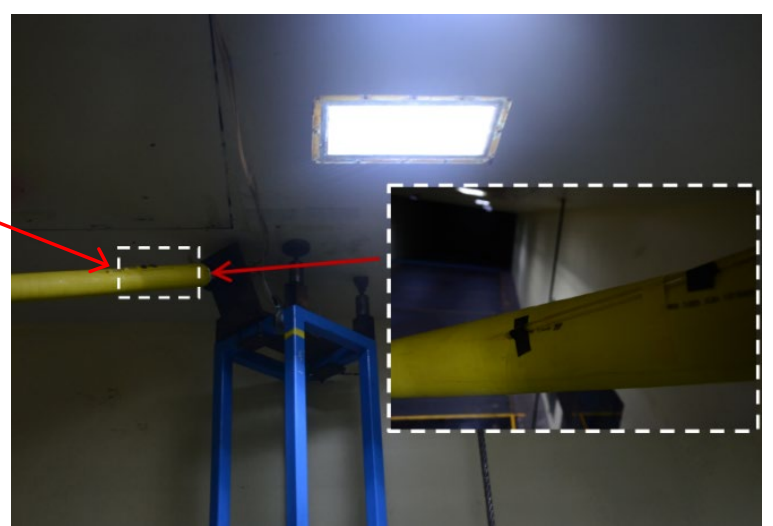

(b)

Figure 2. Cable model configuration: (a) spatial position of the inclined cable and (b) guiding water on the upper end of the cable to generate upper rivulet, thus exciting RWIVs.

\section{Experiment results}

\subsection{Multi-modal VIVs of the flexible cable}

The root-mean-square (RMS) values of the acceleration and vibration displacements (both in-plane and out-of-plane) of the flexible cable model are collected and plotted against incoming wind speeds in Fig. 3. The displacement time-histories were obtained by integrating the recorded accelerations. The amplitudes, $(A)$, in the present study, were normalised by dividing by the cable diameter, $(D)$. When the wind speed gradually increased from $1.33 \mathrm{~m} / \mathrm{s}$, the dry cable remained initially stable. As the wind speed exceeded $1.67 \mathrm{~m} / \mathrm{s}$, cable vibrations could be observed. Afterwards, the cable vibrations became larger with the increase of wind speed. When the wind speed reached $1.92 \mathrm{~m} / \mathrm{s}$, the vibration amplitudes obtained a local maximum. Then, the cable vibrations witnessed a downward trend, whereas the wind speed continuously increased. At an incoming wind speed of $2.71 \mathrm{~m} / \mathrm{s}$, the vibration amplitudes reached the local minimum. Then, the first-mode frequency lock-in ended, and the second-mode lock-in developed. 
Therefore, the first-mode VIVs occurred in the wind speed range of $1.67-2.71 \mathrm{~m} / \mathrm{s}$. Additionally, the in-plane vibrations were much more profound than the out-of-plane vibrations for each test case, which is consistent with the field monitoring results of Zuo et al. [12] and Zuo and Jones [13]. As the wind speed continued to increase, second and third mode VIVs occurred. The phenomena of higher modes VIVs were like that of the first mode, but the frequency lock-in range was found to expand gradually. Moreover, the local maximum of the cable vibrations for the second- and third-mode VIVs were found at an incoming wind speed of 3.42 and $5.42 \mathrm{~m} / \mathrm{s}$, respectively. Therefore, three test cases at the incoming wind speeds of $1.92,3.42$ and $5.42 \mathrm{~m} / \mathrm{s}$ were chosen to analyse the multi-modal VIVs.

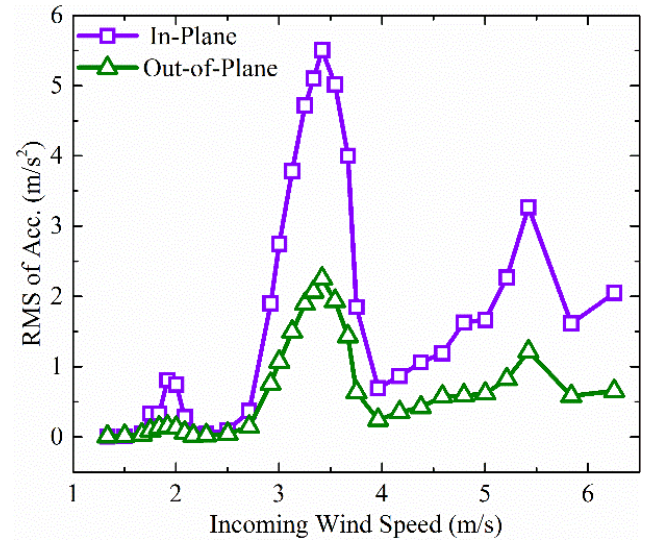

(a)

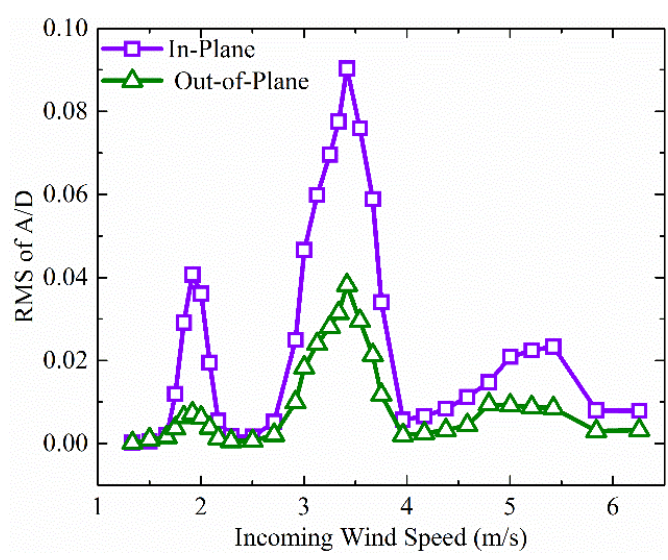

(b)

Figure 3. RMS of accelerations (a) and the normalised vibration amplitudes (b) of the inclined cable with the increase of wind speed.

At the incoming wind speed of $1.92 \mathrm{~m} / \mathrm{s}$, the flexible cable model experienced steady VIVs. As seen in Fig. 4, the vibration amplitudes experienced no significant change over time. The in-plane vibration amplitudes were much larger than the out-of-plane vibration amplitudes. The in-plane vibrations and out-of-plane vibrations were nearly 
anti-phase, with a phase difference $(\phi)$ of $158.2^{\circ}$, as shown in Fig. 4(c). Moreover, the frequency spectrum of the in-plane and out-of-plane vibrations were both identified by fast Fourier transform (FFT) analysis, as shown in Fig. 5(a). The dominant frequency of the vortex VIVs was $2.30 \mathrm{~Hz}$, both in-plane and out-of-plane, but the natural out-ofplane frequency of the cable model was slightly smaller than its in-plane counterpart, as presented in Table. 1. If the in-plane vibration frequency is consistent with the outof-plane vibration frequency, the cable's motion trajectory will be an inclined 'I' shape or an ellipse, decided by the phase difference, $(\phi)$, between the in-plane and out-ofplane vibrations. However, if the out-of-plane vibration frequency is twice the in-plane vibration frequency, the motion trajectory of the cable will be a figure-eight shape. In this case, the motion trajectory of the cable undergoing the first mode VIVs was characterised by an ellipse, as shown in Fig. 5(b), because its frequencies in two directions were coupled (Fig. 5(a)).

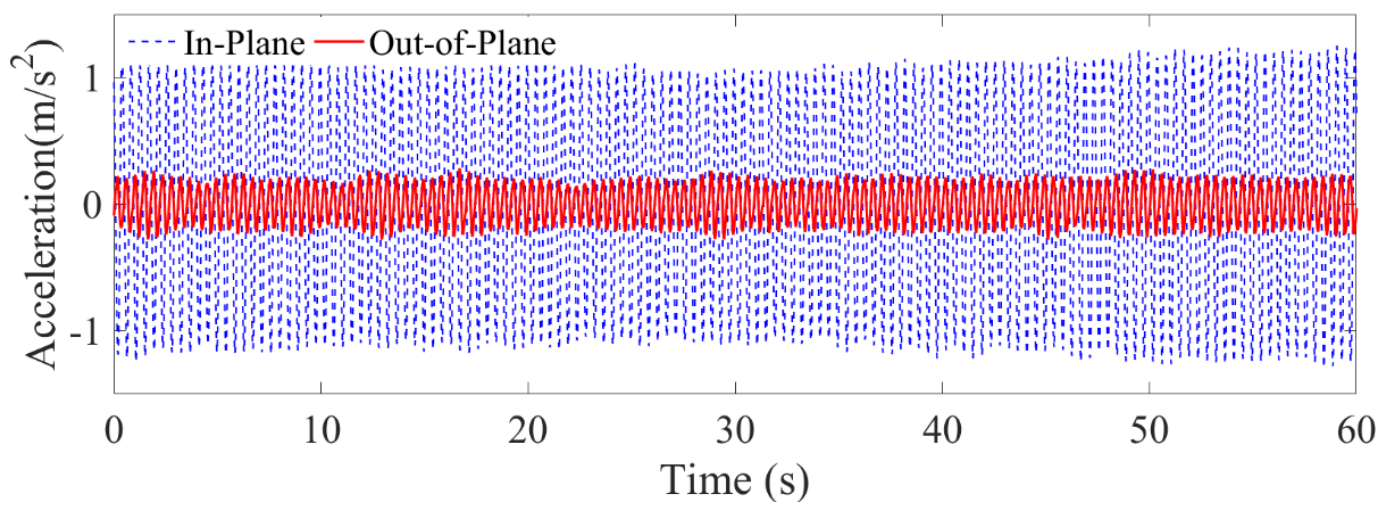

(a) 


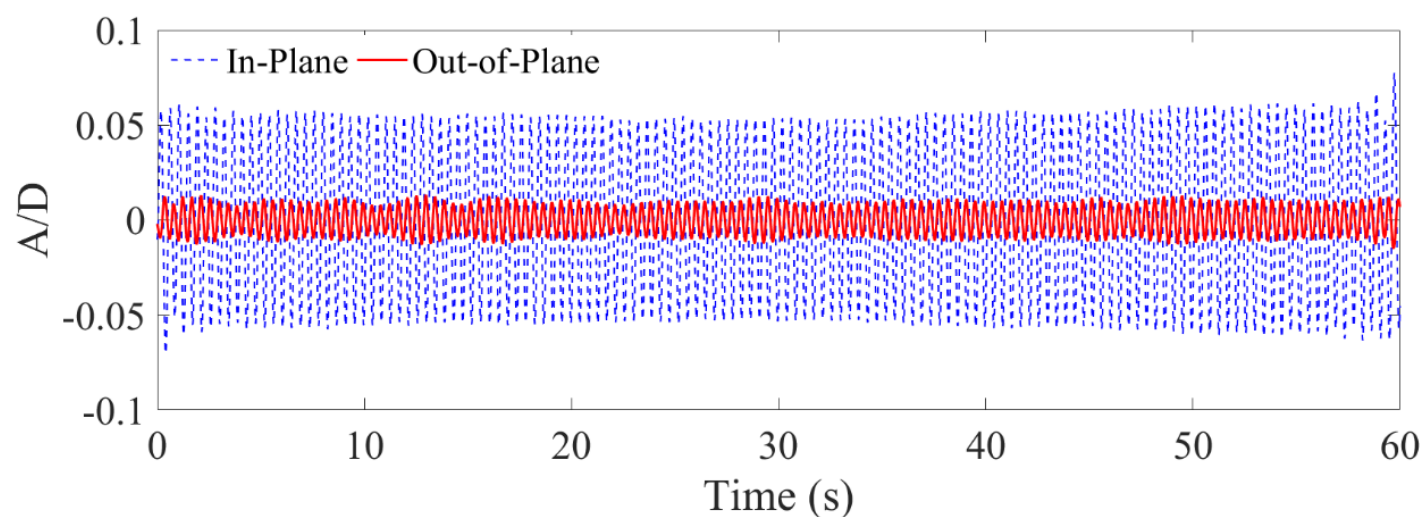

(b)

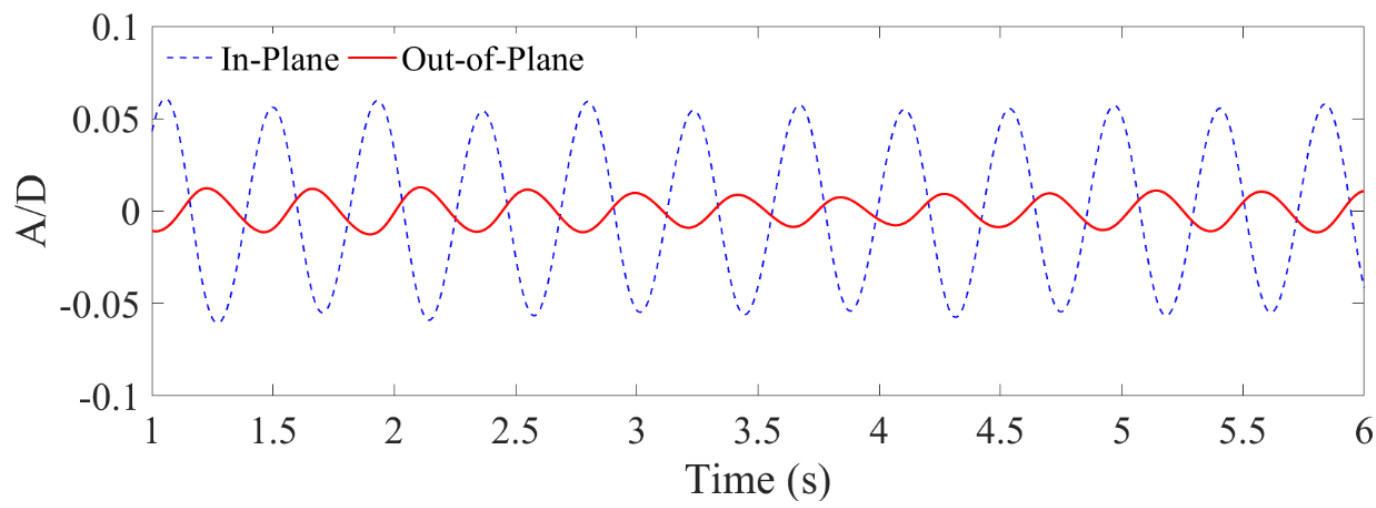

(c)

Figure 4. Accelerations (a) and the normalised displacement responses (b and c) of the first mode VIVs at $1.92 \mathrm{~m} / \mathrm{s}$.

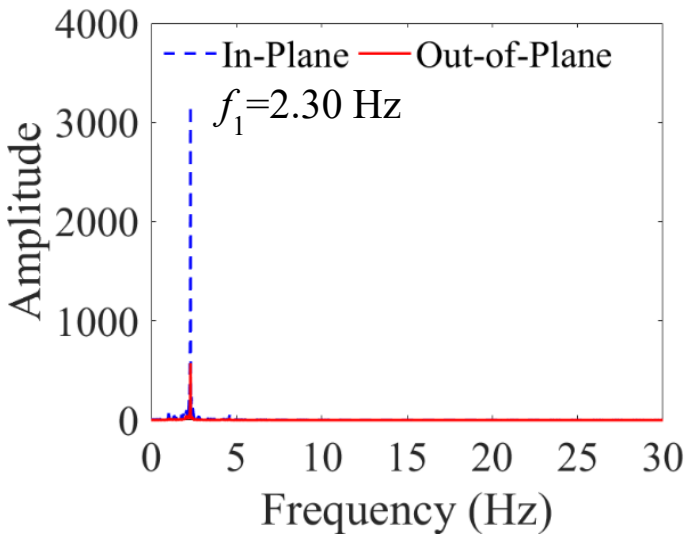

(a)

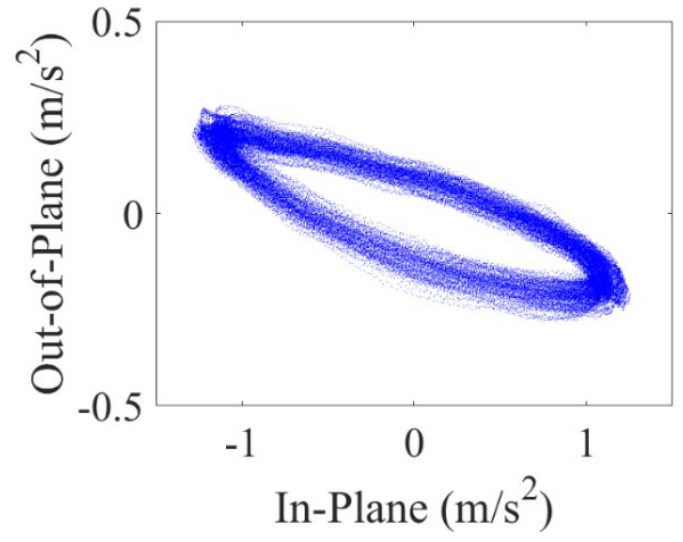

(b)

Figure 5. Frequency spectrum (a) and motion trajectory (b) of the first mode VIVs.

The second- and third-mode VIVs were observed at the incoming wind speeds of 3.42 and $5.42 \mathrm{~m} / \mathrm{s}$, respectively. The experiment results are presented in Figs. 6-9. While 
they took place at higher wind speeds and higher frequency lock-in ranges, the phenomena of higher-mode VIVs exhibited substantial similarities to first-mode VIVs, including the steady vibration amplitudes, frequency lock-in and the elliptical motion trajectories. Additionally, the phase differences, $(\phi)$, of the second and third mode VIVs were $1.43^{\circ}$ and $3.64^{\circ}$, respectively, indicating in-phase VIVs at wind speeds of 3.42 and $5.42 \mathrm{~m} / \mathrm{s}$.

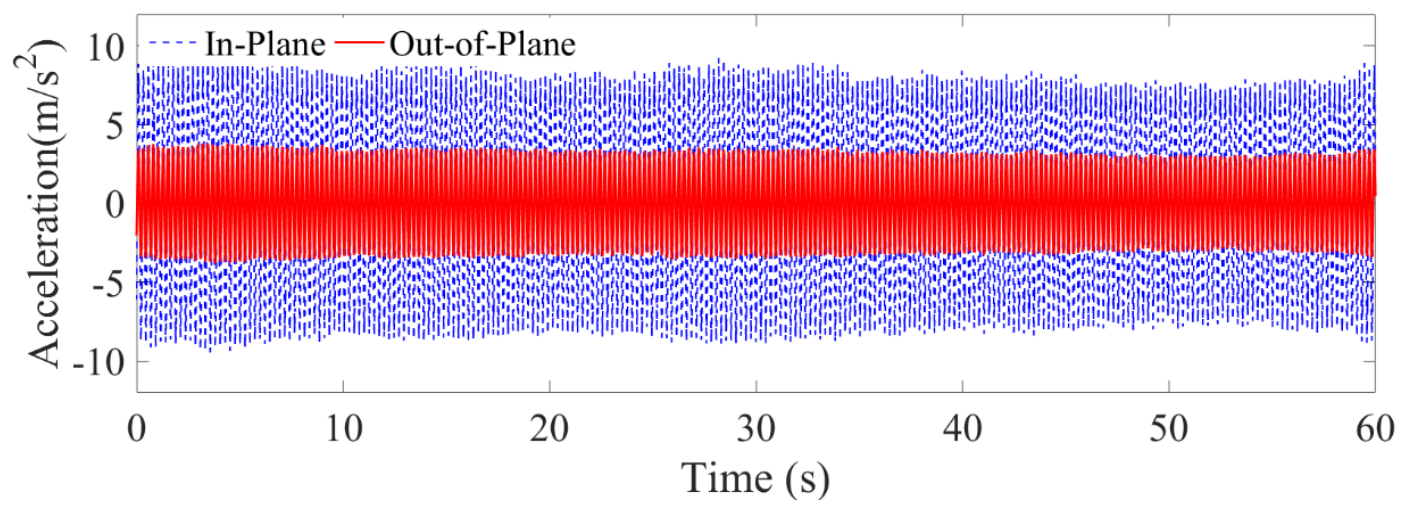

(a)

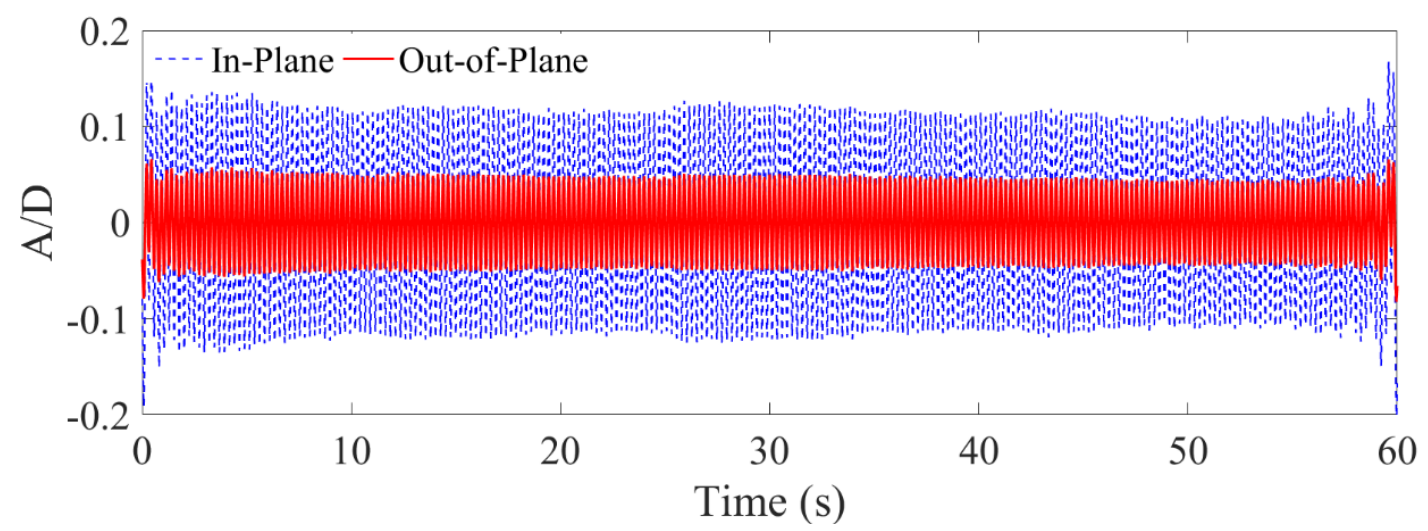

(b) 


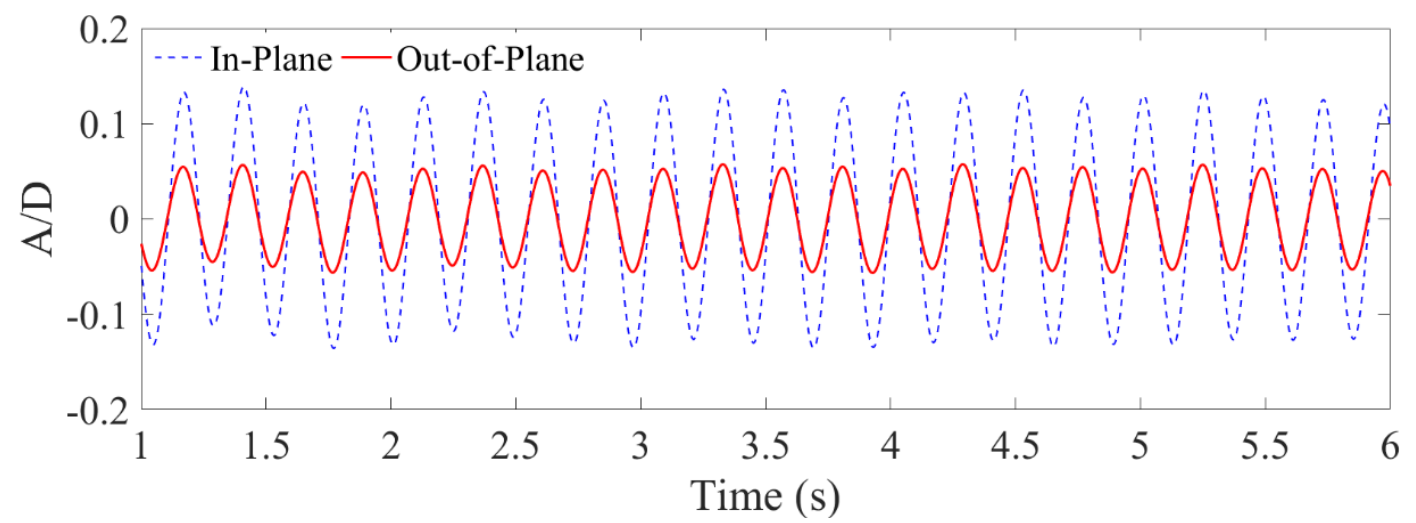

(c)

Figure 6. Accelerations (a) and the normalised displacement responses (b and c) of the second mode VIVs at $3.42 \mathrm{~m} / \mathrm{s}$.

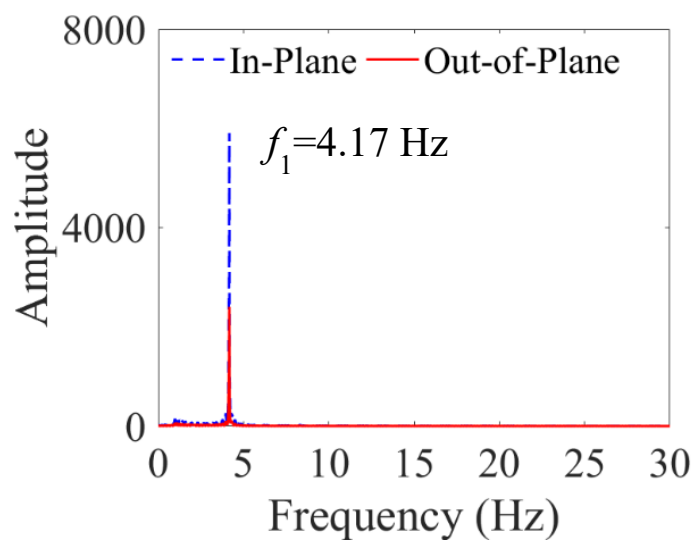

(a)

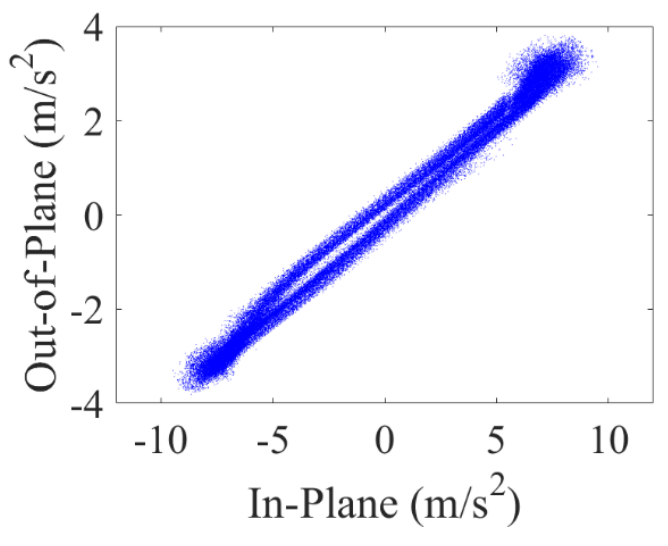

(b)

Figure 7. Frequency spectrum (a) and motion trajectory (b) of the second mode VIVs.

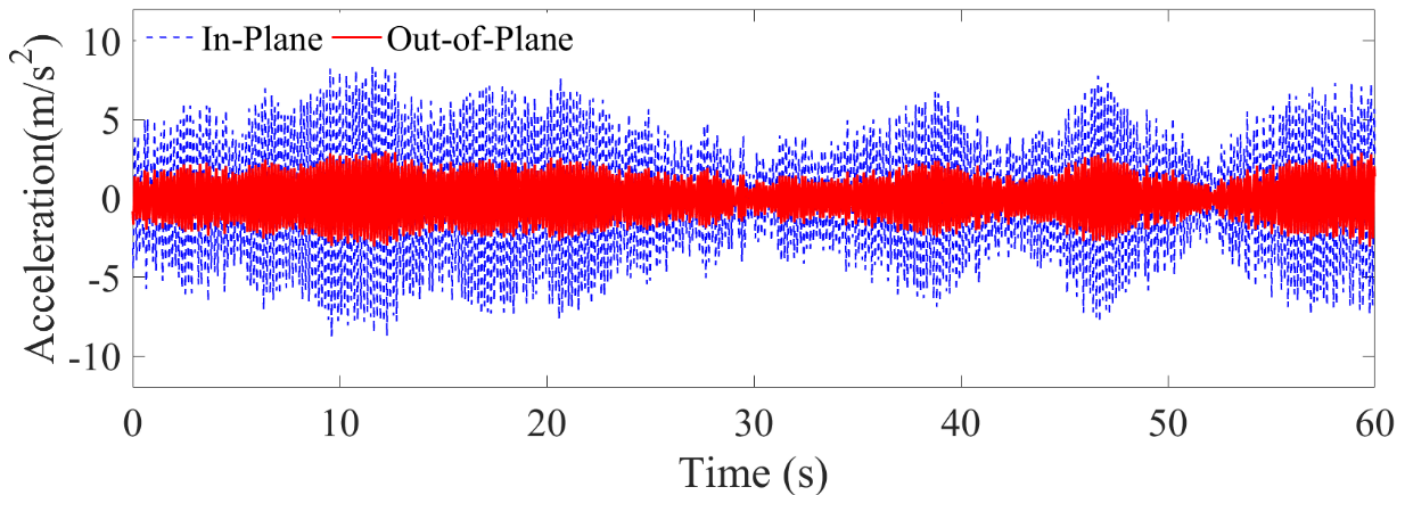

(a) 


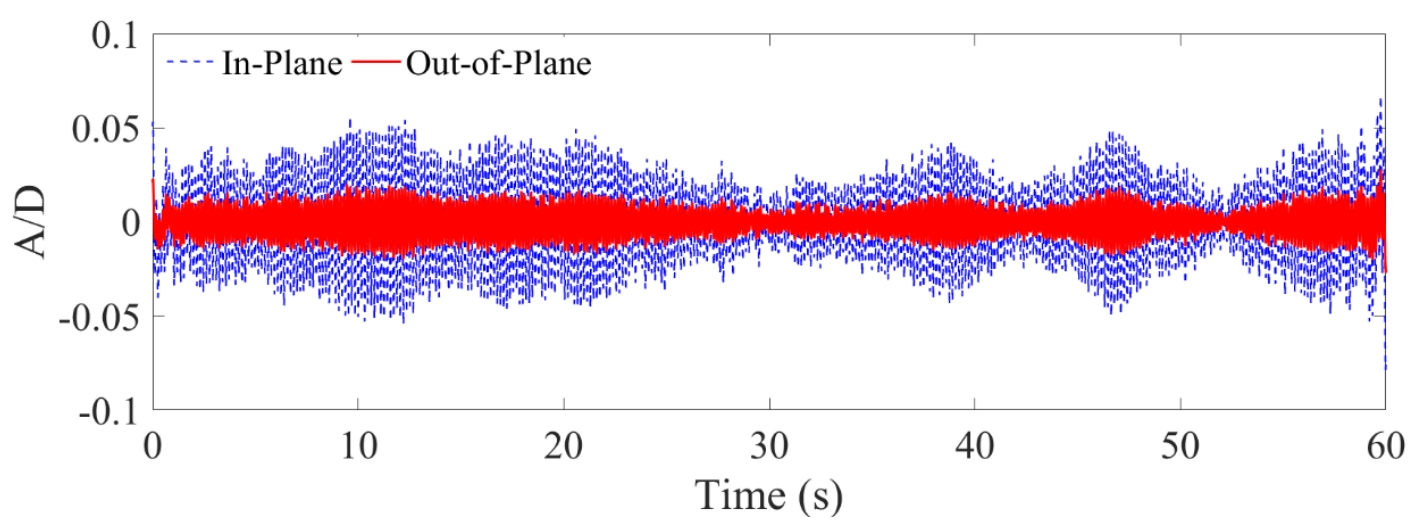

(b)

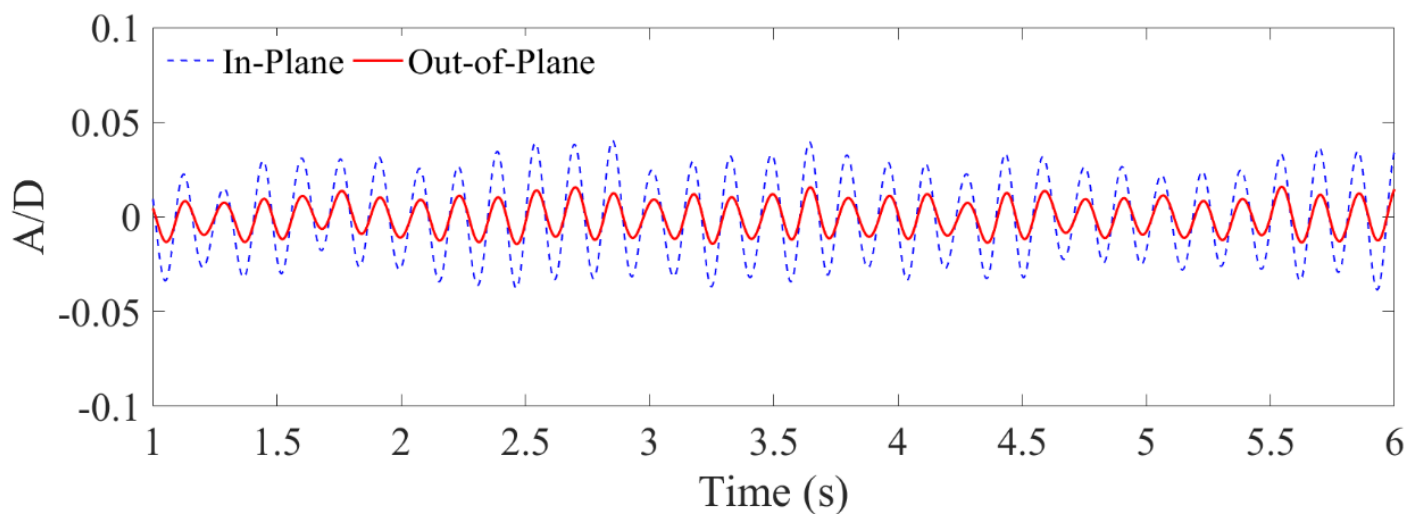

(c)

Figure 8. Accelerations (a) and normalised displacement responses (b and c) of the third mode VIVs at $5.42 \mathrm{~m} / \mathrm{s}$.

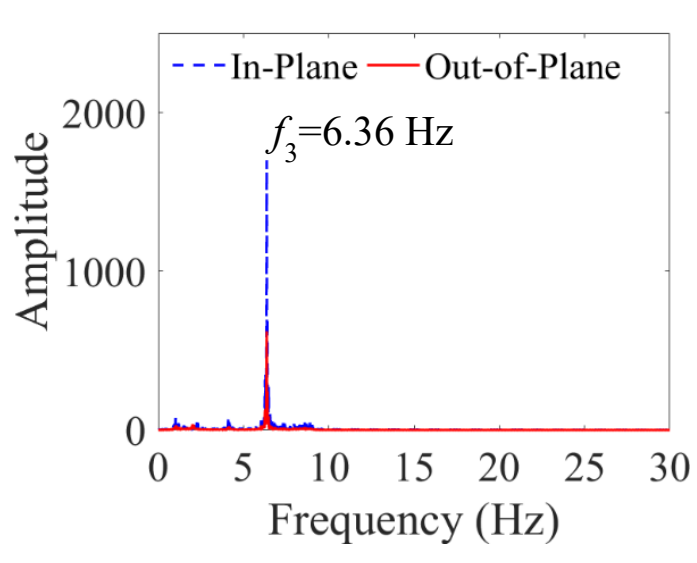

(a)

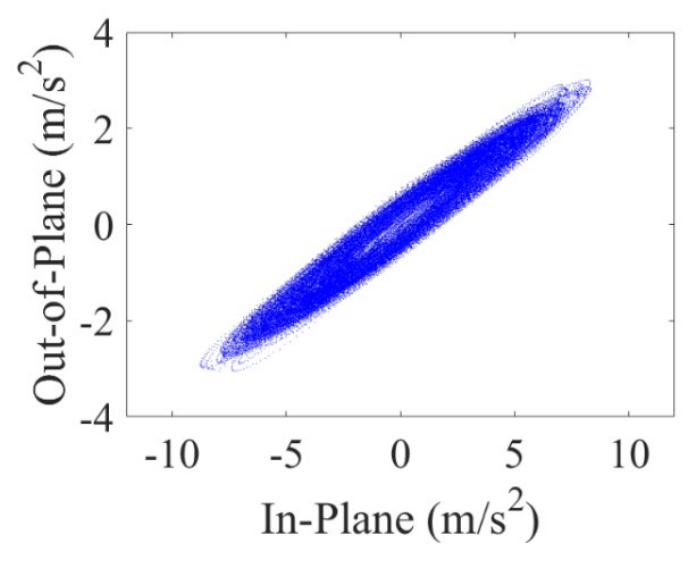

(b)

Figure 9. Frequency spectrum (a) and motion trajectory (b) of the third-mode VIVs.

\subsection{Multi-modal RWIVs of the flexible cable}


The wind tunnel tests for RWIVs started with a wind speed of $7.92 \mathrm{~m} / \mathrm{s}$ and gradually increased in steps of $0.42 \mathrm{~m} / \mathrm{s}$. First, the upper water rivulet failed to form at low wind speed because it dropped or slipped down to the lower surface of the cable model. Consequently, the wind-induced vibrations of the flexible cable were observed to be minimal, and no RWIVs signal was recorded by the accelerometers. At a wind speed of $8.76 \mathrm{~m} / \mathrm{s}$, some water rivulets intermittently climbed up the cable surface. However, no continuous upper rivulet was observed along the cable, and thus no RWIVs were generated. However, when the incoming wind speed shifted from $10.01 \mathrm{~m} / \mathrm{s}$ to 10.43 $\mathrm{m} / \mathrm{s}$, an upper rivulet gradually formed along the entire cable, moving periodically around the cable surface. The lower rivulet was clear, but nearly static. Meanwhile, a violent cable vibration was easily detected. Therefore, multi-modal behaviours of RWIVs in a stay cable were successfully reproduced in our wind tunnel tests. RWIVs at this wind speed were dominated by its first vibration-shape mode. As the wind speed continued to increase, the amplitudes of RWIVs gradually enhanced. As the wind speed changed from $13.76 \mathrm{~m} / \mathrm{s}$ to $14.18 \mathrm{~m} / \mathrm{s}$, the vibration amplitudes were found to decrease while remaining notable, as shown in Fig. 10(a). In this case, the mode shape indicates that the cable vibration was dominated by the second mode because the node was nearly fixed at $L / 2$. When the wind speed increased to $14.60 \mathrm{~m} / \mathrm{s}$, the dominant vibration mode was changed to its third mode, as revealed by the frequency analysis in Fig. 10(b). Then, the cable vibrated at its third mode with its wind speed getting higher. When the incoming wind speed was in the range of $14.18-14.60 \mathrm{~m} / \mathrm{s}$, the amplitudes of cable vibrations diverged because of the mode-switch scenario ([27, 28]). During RWIVs, the 
in-plane vibration amplitudes were much larger than the out-of-plane vibrations, as can be seen in Fig. 10(a). This agrees with the field monitoring results by Zuo et al. [12] and Zuo and Jones [13]. In our study, three different test cases, at incoming wind speeds of $11.26,14.18$ and $15.43 \mathrm{~m} / \mathrm{s}$, were chosen to analyse the first-, second- and thirdmode-dominated RWIVs, respectively.

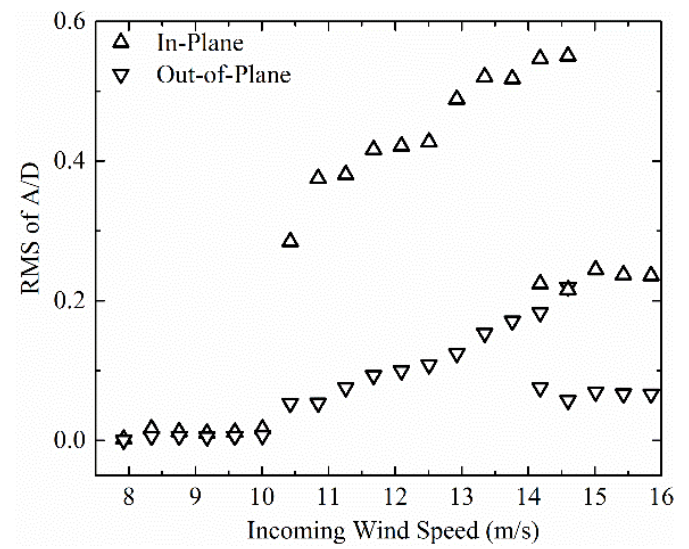

(a)

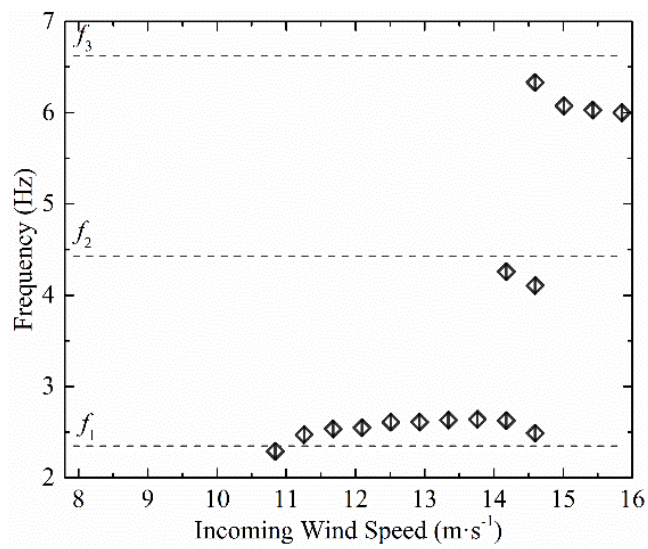

(b)

Figure 10. RMS of the normalised vibration amplitudes (a) and frequency evolution (b) of the inclined cable under the joint action of wind and rain. The dashed lines denote the modal frequencies of the cable measured in still air.

The acceleration signals of the flexible cable suffering from RWIV at the incoming wind speed of $11.26 \mathrm{~m} / \mathrm{s}$ are presented in Fig. 11(a). The cable vibrations were profound, especially in the in-plane direction. This is consistent with the field monitoring results reported by $\mathrm{Ni}$ et al. [11], where they found that the in-plane acceleration was more prominent than the out-of-plane acceleration during RWIV. By integrating the accelerations, the normalised displacements of the in-plane and out-of-plane vibrations are obtained and shown in Fig. 11(b). Within a period of $60 \mathrm{~s}$, the flexible cable experienced steady RWIVs. By performing an FFT analysis, the frequency spectrum of the cable vibrations were identified to plot Fig. 12. It is clear that the RWIVs in this 
case were mainly dominated by the first mode.

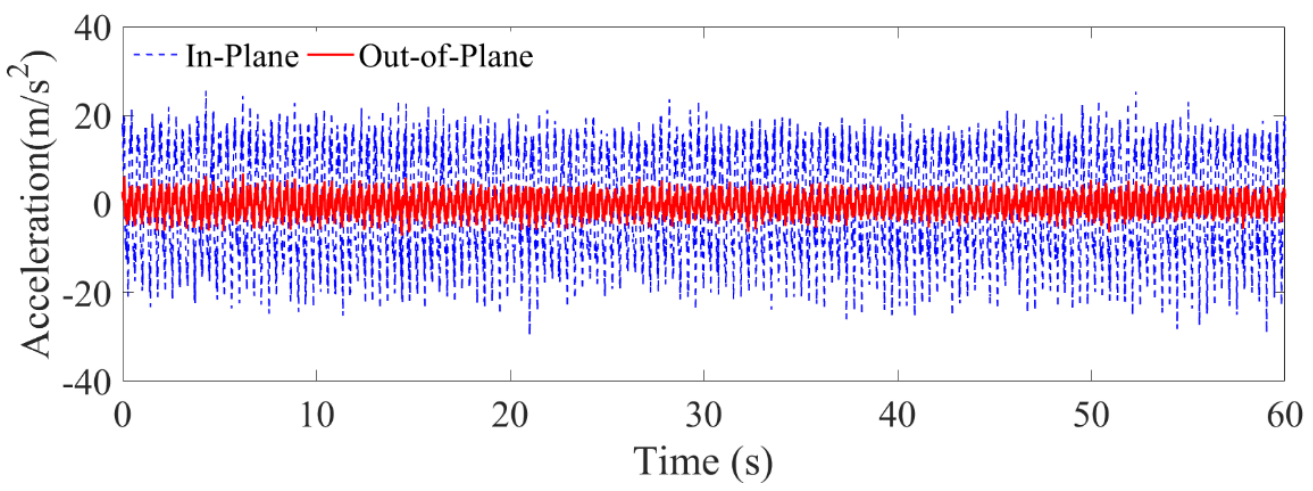

(a)

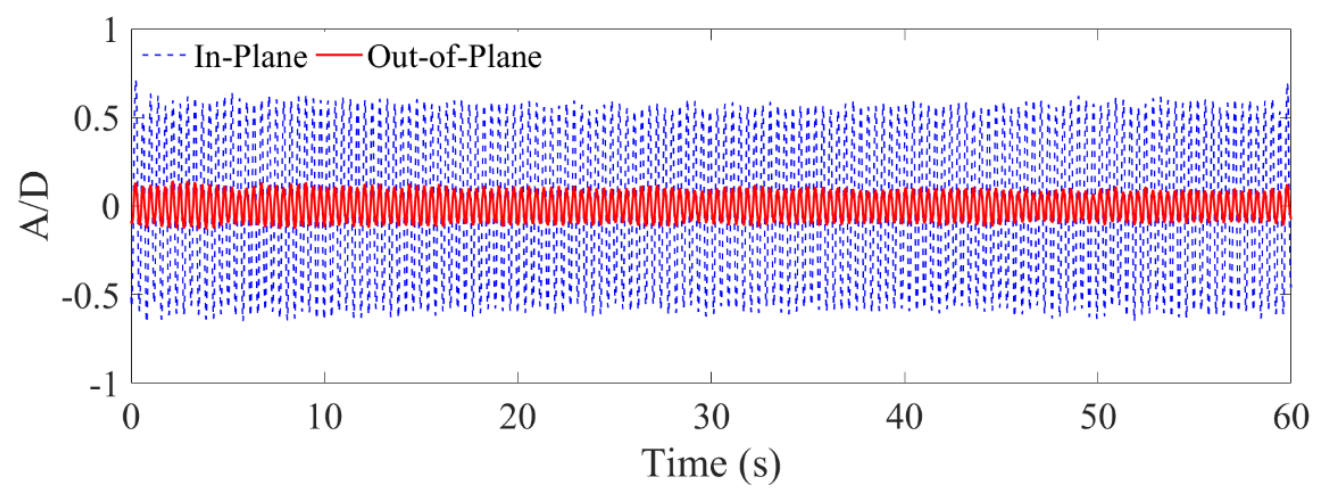

(b)

Figure 11. Accelerations (a) and the normalised displacement responses (b) of the first mode RWIVs at $11.26 \mathrm{~m} / \mathrm{s}$.

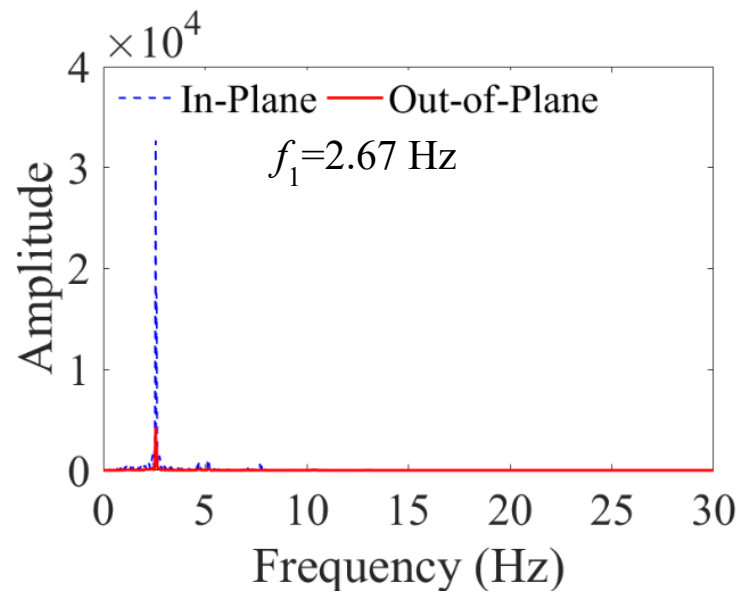

Figure 12. Frequency spectrum of the first mode RWIVs.

As the wind speed increased to $14.18 \mathrm{~m} / \mathrm{s}$, second-mode RWIVs occurred. The acceleration signals of the flexible cable, in this case, are plotted in Fig. 13(a). Like 
what happened in the first-mode RWIVs, the in-plane cable vibrations were larger than the out-of-plane vibrations. By integrating accelerations, the displacements of in-plane and out-of-plane vibrations were obtained and shown in Fig. 13(b). The peak in-plane amplitude of the RWIVs, measured at $L / 6$ knots, was about 0.3 times the cable diameter. The frequency spectrum of the cable vibrations is plotted in Fig. 14 to demonstrate that the cable experienced second-mode RWIVs.

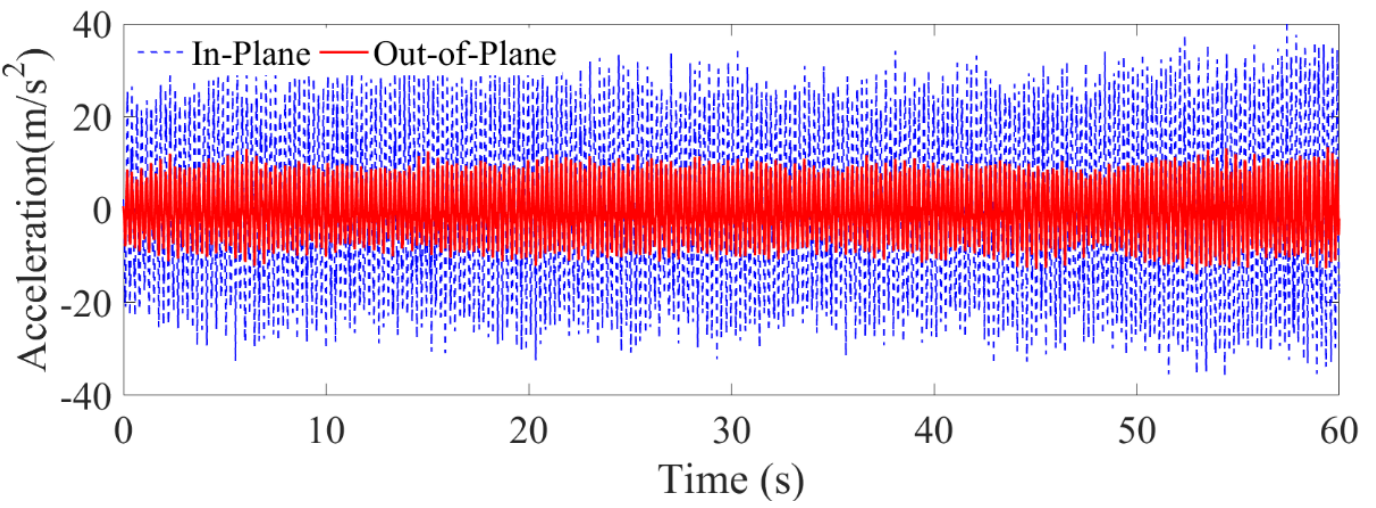

(a) 


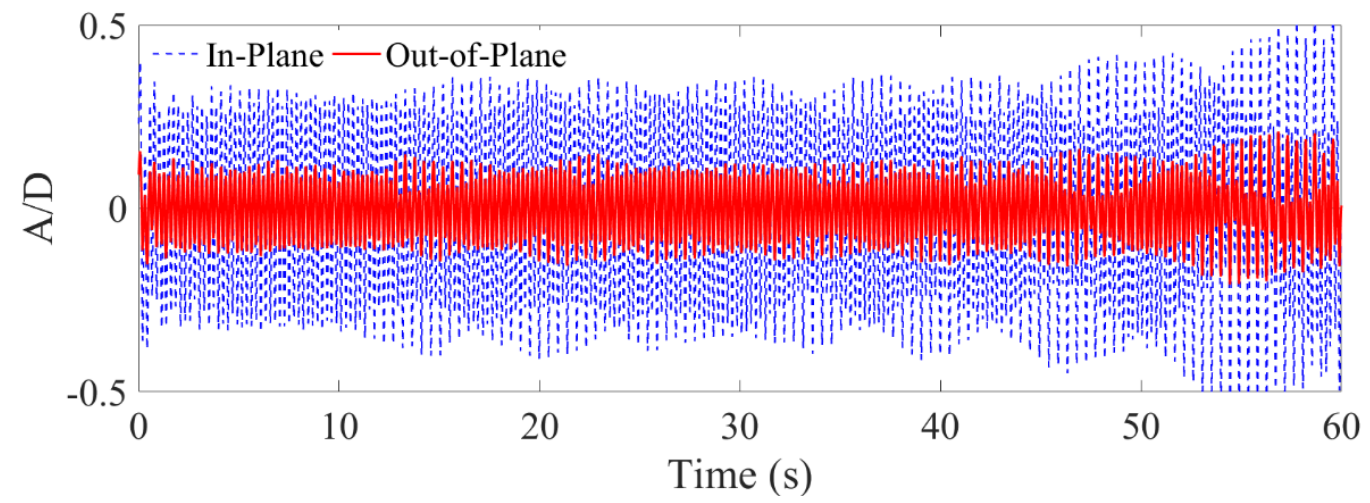

(b)

Figure 13. Accelerations (a) and the normalised displacement responses (b) of the second mode RWIVs at $14.18 \mathrm{~m} / \mathrm{s}$.

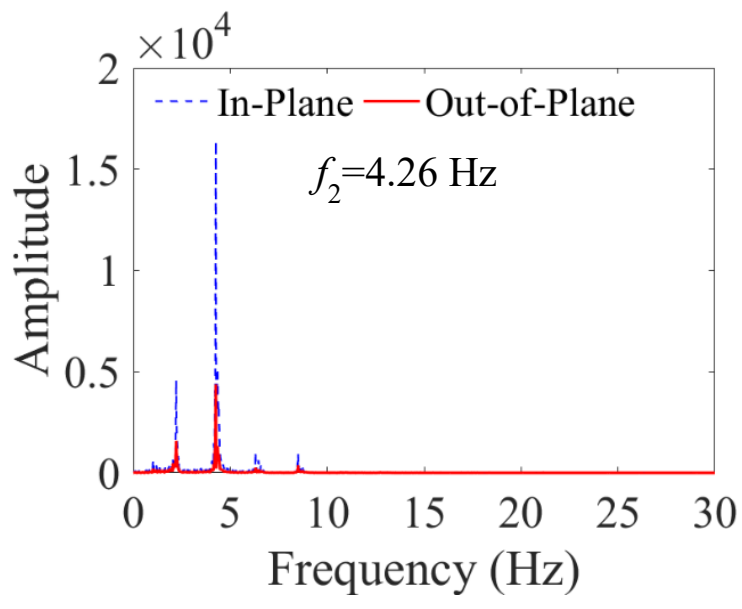

Figure 14. Frequency spectrum of the second-mode RWIVs.

Figure. 15 illustrates the vibration time-histories of the third-mode-dominated RWIVs at a wind speed of $15.43 \mathrm{~m} / \mathrm{s}$, and Fig. 16 presents their frequency spectrum. The phenomenon is like the first and second RWIVs, but with a higher order frequency. The higher-mode RWIVs tend to occur at higher wind speed. These characteristics of RWIVs are similar to those of the multi-modal VIVs. It is believed that a higher wind speed contributes to a larger vortex shedding frequency from the cable model, and that the wake frequency can couple with the higher mode of the cable's frequency. This 
wake-cable synchronization, which exhibits similarities to the lock-in phenomenon of VIVs, resulted in large-amplitude RWIVs.

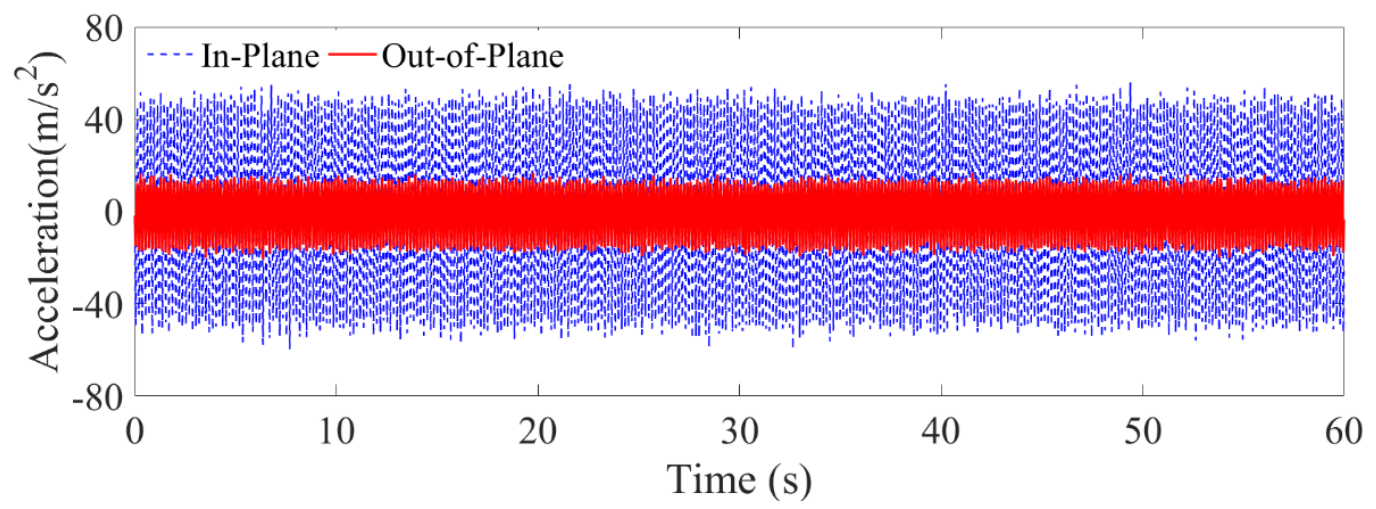

(a)

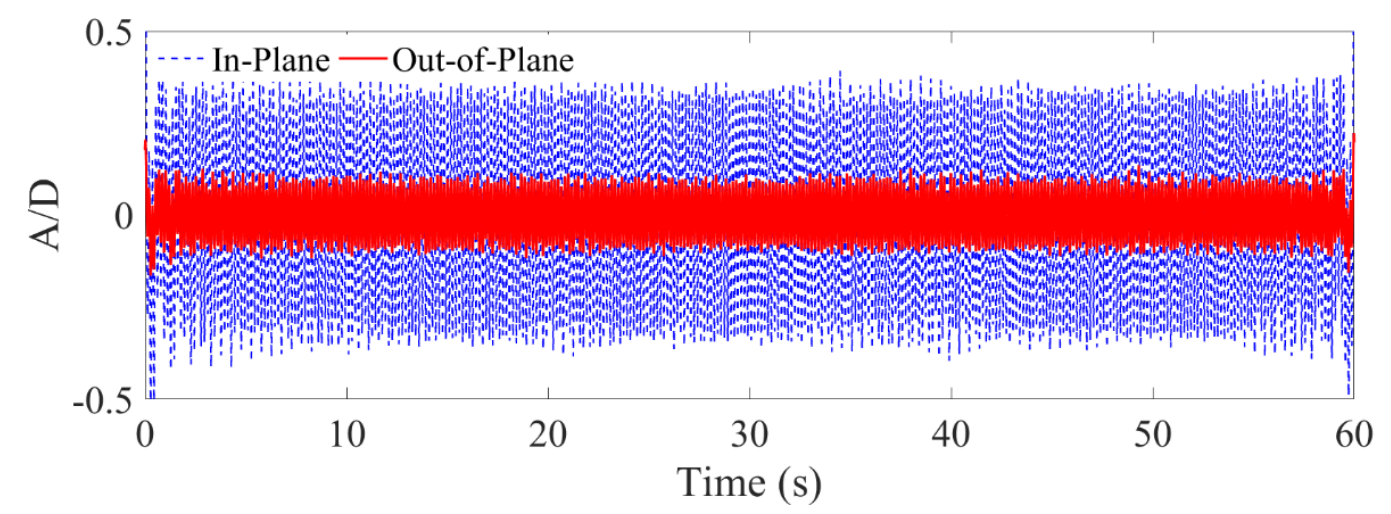

(b)

Figure 15. Accelerations (a) and normalised displacement responses (b) of thirdmode RWIVs at $15.43 \mathrm{~m} / \mathrm{s}$. 


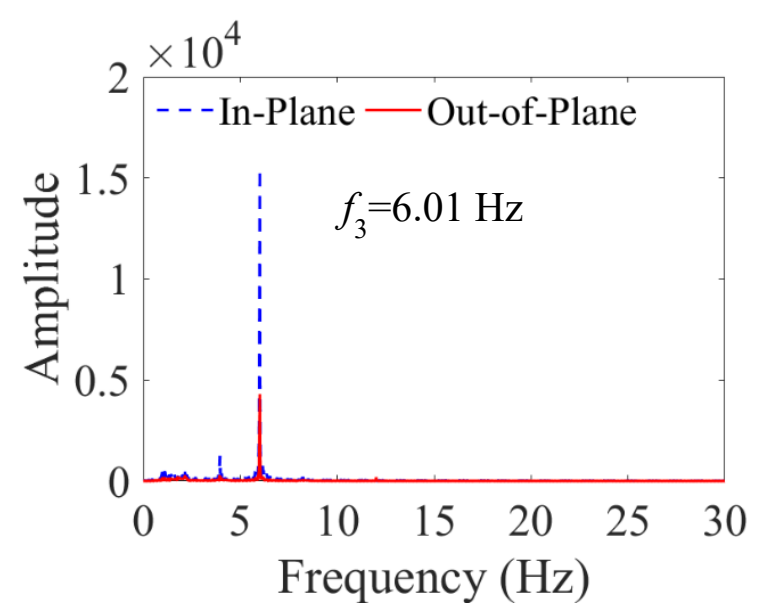

Figure 16. Frequency spectrum of third-mode RWIVs.

Unlike VIVs, the dominant frequencies of RWIVs were found to deviate from the modal frequencies of the dry cable in still air. During our experimental campaign, before and after wind tunnel tests, we conducted two free vibration tests for each case to ensure that no tension slack occurred in the steel wire ropes. Thus, the frequency deviation during experiments was caused by the joint action of changing tension forces and the added mass of water. According to Irvine [30], the vibration frequency of a cable can be described as

$$
f=\frac{n}{2 L} \sqrt{\frac{\Theta}{m_{S}}},
$$

where $n$ denotes the $n^{\text {th }}$ mode, $\Theta$ is the axial tension and $m_{S}$ is the cable mass. Owing to the existence of lower and upper rivulets, the added mass always leads to a decrease of the vibration frequency. With a larger vibration amplitude, the effects of the added mass are minimal because only a small amount of water is retained on the cable surface (i.e., the rivulets become thin). Conversely, when the vibration amplitude is small, the effects of added mass become more profound. However, a larger vibration amplitude contributes to an increase of axial tension force and, therefore, an increase 
of the vibration frequency, as can be derived from Eq. (1). This observation has also been confirmed and reported by previous studies (see Fig.11 (a) in Ref. [28]).

In first-mode RWIVs, the vibration amplitude was large, thus the tension force was substantially larger and the added mass was small. Thus, the vibration frequency increased by $0.36 \mathrm{~Hz}$. However, when the flexible cable experienced third-mode RWIVs, the vibration amplitudes became smaller and more water was retained on the cable surface (i.e., the rivulets became thick). Thus, the effects of added mass became dominant, whereas the effect of the increased axial tension force greatly shrunk, resulting in a slightly smaller frequency than that of the dry cable in still air. In the case of the second-mode RWIVs, the effects of the two factors roughly overcame each other. Thus, the vibrations frequencies were close to that of the dry cable.

\subsection{RWIVs excited by locally distributed rivulets}

As shown in the field monitoring results reported by Zuo et al. [12], in-situ RWIVs often exhibit multiple modes. Besides the three-dimensional incoming airflow, the distribution of upper rivulets is also suspected to play a crucial role in the occurrence of multi-modal RWIVs. During bridge engineering, stay cables are usually as long as $100 \mathrm{~m}$. Therefore, rivulets cannot possibly be formed along the entire cable length. Inspired by this, we investigated the RWIVs excited by locally distributed upper rivulets. The locally distributed upper rivulets were generated by changing the position of the water supply and using a pipe to prevent rivulets from forming along the entire cable length. We designed four types of locally distributed upper rivulets, as shown in Fig. 
17. The upper rivulet was guided and restricted to form in the middle $0.5 \mathrm{~L}$ ( $\mathrm{L}$ is the cable length), in the upper $0.33 \mathrm{~L}$, in the upper $0.50 \mathrm{~L}$ or in the upper $0.66 \mathrm{~L}$. These test cases are denoted as LR1, LR2, LR3 and LR4, respectively. During the wind tunnel tests, the wind speed also increased, as described in Section 3.2. To facilitate comparisons, more attention was paid to the cases where the incoming wind speed was $\mathrm{V}=11.26 \mathrm{~m} / \mathrm{s}$. We recall that at this wind speed, RWIVs are dominated by their first mode if the rivulet can be formed along the entire cable. This incoming wind speed is also much lower than $14.18 \mathrm{~m} / \mathrm{s}$, the onset wind speed in the present experimental setup for the occurrence of second-mode RWIVs.
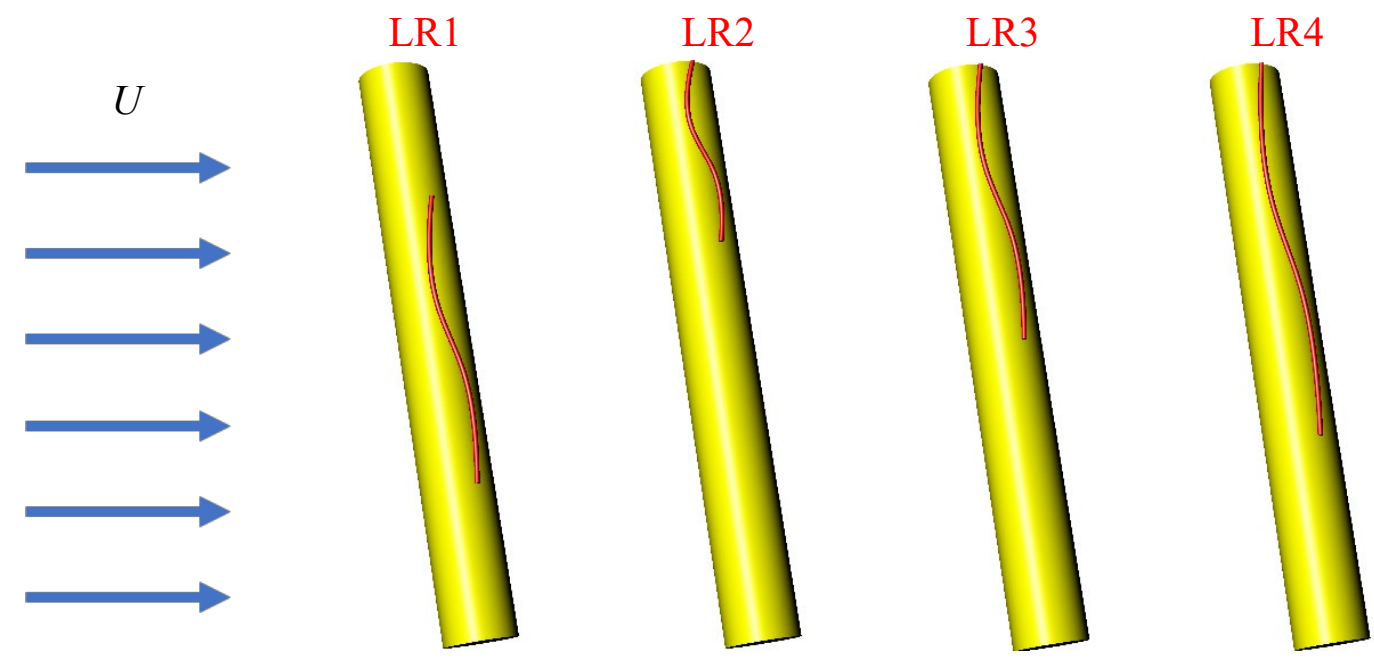

Figure 17. Sketch of the locally distributed upper rivulets.

RWIV responses of the cable with different guided rivulets at an incoming wind speed $\mathrm{V}=11.26 \mathrm{~m} / \mathrm{s}$ are measured and presented in Fig. 18. For the test case of LR1 (i.e., the upper rivulet is restricted to form in the middle L/2), the RWIV is identified as the first mode, as shown in Fig. 18. However, the vibration amplitudes were smaller than those in Fig. 11, when the upper rivulet was formed along the entire cable. Additionally, when 
the upper rivulet was guided to form in the upper L/3 and was prevented from slipping down to the lower span of the cable, for test case LR2, the RWIVs were identified as second-mode at the same wind speed. When the rivulet formation length was limited to the upper L/2, for test case LR3, the RWIVs were also identified as second-mode, but the vibration amplitudes were notably larger than those of test case LR2. Furthermore, because the upper rivulet could form in the upper $2 \mathrm{~L} / 3$, for test case LR4, RWIVs of the flexible cable exhibited multiple modes. By performing an FFT analysis to identify the frequency components, the cable vibrations were found to be jointly dominated by the first and second modes, as shown in Fig. 18.

In the previous study of $\mathrm{Ni}$ et al. [11], they reported that the overall dominant mode of the RWIVs was the third mode, but the dominant mode can differ for various response segments. In our experimental study, whereas the cable length was limited and much smaller than real cables, the RWIVs with different modes and amplitudes were successfully reproduced by guiding differently and locally distributed upper rivulets. This implies that the local distributions of upper rivulets contributed to exciting the multi-modal RWIVs, providing another possible explanation for the field observation of the RWIVs of real cables often exhibiting multiple modes.
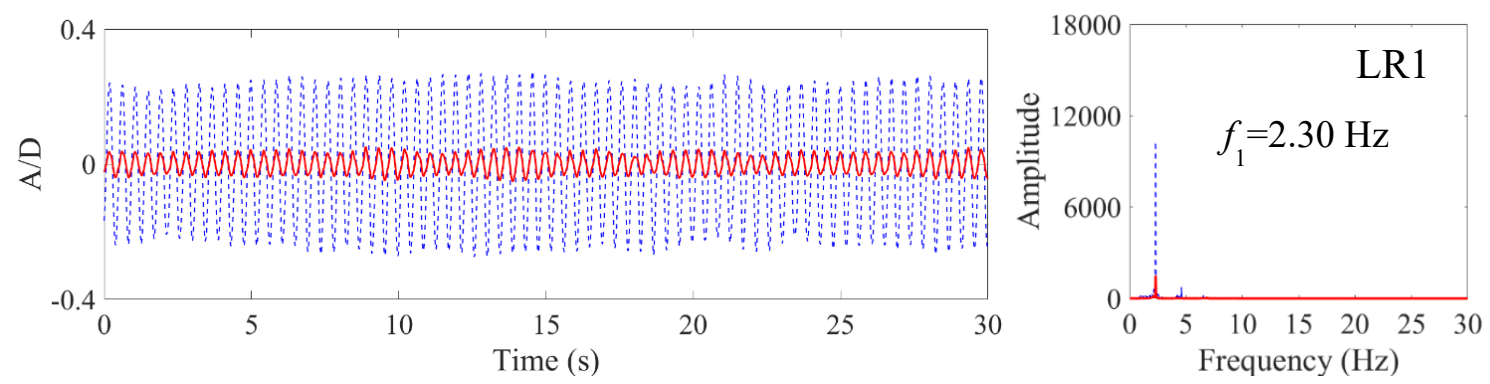

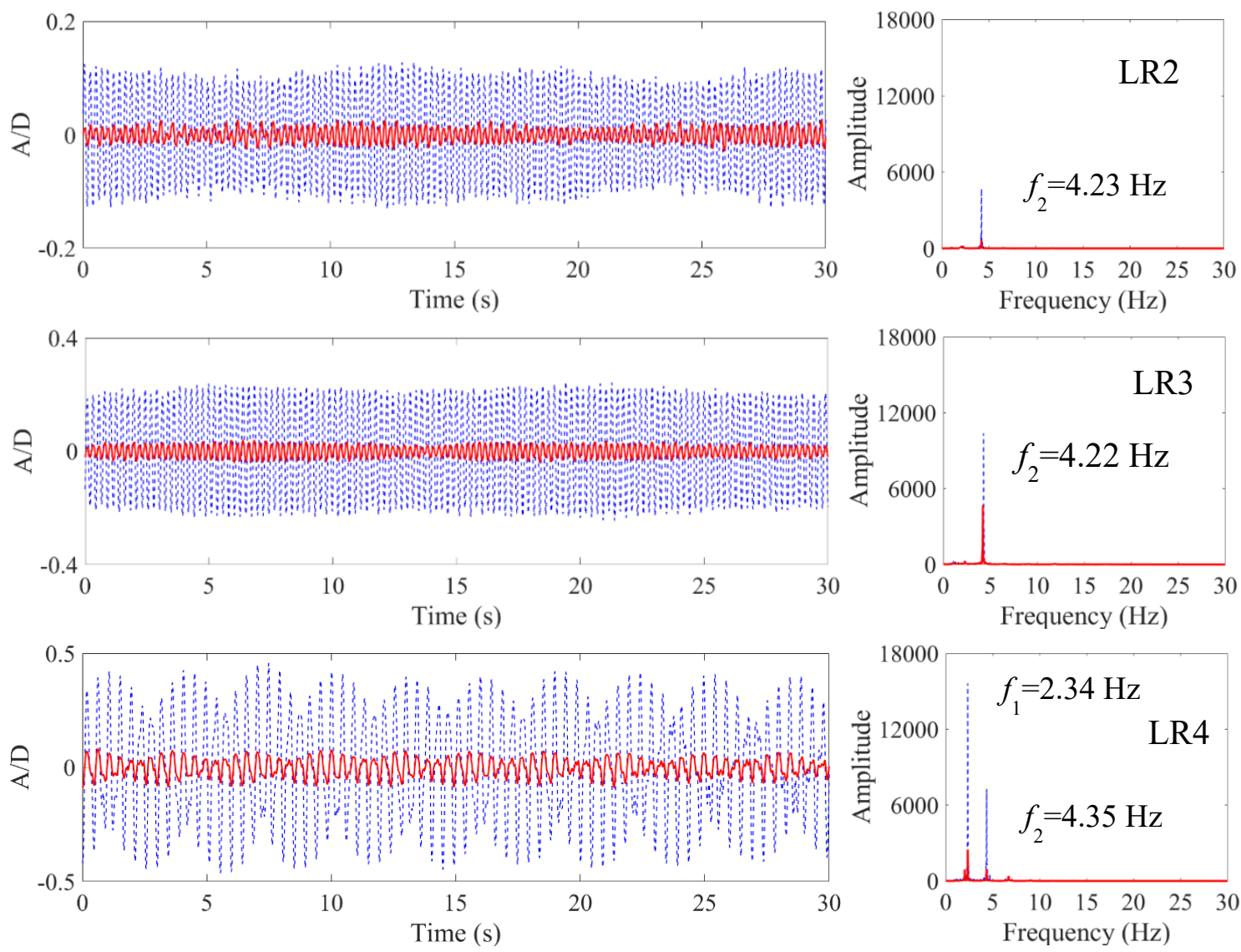

Figure 18. The normalised displacement responses (left) and frequency spectrum (right) of the flexible cable under different rivulet distributions. In every subplot, the bluedashed line denotes in-plane signals and the red-solid line denotes the out-of-plane signals.

\subsection{Discussion}

According to the research findings of Matsumoto et al. [24], RWIV was explained as a VIV, but at a high reduced velocity region. In their wind tunnel tests, they found two dominant frequency components: one for Kármán vortex shedding and another with a much lower frequency. The latter was the driving force of RWIV. Moreover, as reported by Zuo et al. [12], substantial similarities exist between VIVs and RWIVs with in-situ field monitoring, perhaps owing to a vortex-induced excitation that is different from conventional Kármán vortex shedding. A low-frequency vortex, for instance, is a driving factor, as shown by Matsumoto et al. [24]. 
In our study, both the higher-mode VIVs and RWIVs tended to develop at a higher wind speed. For VIVs, the frequency of the vortex shedding from the cable became larger as the wind speed increased and synchronised with higher-mode cable frequencies. A similar excitation scenario of RWIVs was found. Additionally, frequency overlap and mode-switching of VIVs have been reported in experiments and theoretical predictions [27-29]. While they were not observed in the VIVs in our experiments, it is interesting to find the frequency overlap and mode switch phenomena in RWIVs, as shown in Fig.10. Based on these experimental results, we conclude that RWIVs exhibited notable similarities to VIVs, as suggested by Zuo et al. [12] and Matsumoto et al. [24]. This undiscovered connection between the two is the key to disclosing the true excitation mechanism of RWIVs. Apart from increasing wind speeds, locally distributed upper rivulets were also found to play a crucial role in exciting multi-modal RWIVs, even under lower wind speeds. Therefore, increased wind speeds and local upper rivulets are two main sources of multi-modal RWIVs in cables.

\section{Conclusions}

By employing one flexible cable model, we reproduced and investigated multi-modal VIVs and RWIVs in wind tunnel tests. We made progress with the study of multi-modal RWIVs of stay cables with our wind tunnel tests. RWIVs were measured on the same framework as VIVs, and the main conclusions are as follows:

1) When the dry cable model was subjected to uniform airflow, it was found to experience first-, second- and third-mode VIVs with increased wind speeds. The 
phenomena of frequency lock-ins, observed in different modes of VIVs of the flexible cable, were similar.

2) It is feasible to reproduce multi-modal RWIVs in a wind tunnel. The key factors are minimizing the damping ratio, increasing the length and reducing the mass of the cable model. The present study verified that guiding water lines on the cable surface can excite the RWIVs.

3) Large-amplitude RWIVs were excited under the joint action of wind and rain. Higher modes of the RWIVs on a flexible cable tended to develop at higher wind speeds, and the mode-switch phenomenon was observed, whereas the incoming wind speed remained the same.

4) The RWIVs of the flexible cable were found to exhibit different vibration modes and amplitudes, owing to the different distributions of the guided upper rivulet. The experiments suggest that the local distributions of upper rivulet contributed to exciting the multi-modal RWIVs, even under lower wind speeds. Therefore, the effects of locally distributed upper rivulets provided another possible cause for the occurrence of multi-modal RWIVs, generally observed in real cables of cablestayed bridges.

5) RWIVs on flexible cables exhibit similarities to VIVs in wind tunnel tests. Underlying links between the two seemingly different types of wind-induced cable vibrations should not be overlooked; they remain to be explored in future studies.

\section{Acknowledgements}


This study was funded by the National Natural Science Foundation of China (NSFC) through Grants 51378153, 51578188 and 51722805. D. G also acknowledges the financial support from China Scholarship Council (CSC 201606120189).

\section{References}

[1] B.M. Pacheco \& Y. Fujino, 'Keeping cables calm', Civil Eng., 63 (1993) 56.

[2] R.W. Poston, 'Cable-stay conundrum', Civil Eng., 68 (1998) 58-61.

[3] D. Brika \& A. Laneville, 'Vortex-induced vibrations of a long flexible circular cylinder', J. Fluid Mech., 250 (1993) 481-508.

[4] M. Matsumoto, H. Shirato, T. Yagi, M. Goto, S. Sakai \& J. Ohya, 'Field observation of the full-scale wind-induced cable vibration', J. Wind Eng. Ind. Aerod., 91 (2003) 1326.

[5] W. Chen, H. Li, J. Ou \& F. Li, 'Numerical Simulation of Vortex-Induced Vibrations of Inclined Cables under Different Wind Profiles', J. Bridge Eng., 18 (2013) 42-53.

[6] W. Chen, Q. Zhang, H. Li \& H. Hu, 'An experimental investigation on vortex induced vibration of a flexible inclined cable under a shear flow', J. Fluid Struct., 54 (2015) 297-311.

[7] Y. Hikami \& N. Shiraishi, 'Rain-wind induced vibrations of cables stayed bridges', J. Wind Eng. Ind. Aerod., 29 (1988) 409-418.

[8] H. Yamaguchi, 'Analytical study on growth mechanism of rain vibration of cables', J. Wind Eng. Ind. Aerod., 33.1-2 (1990): 73-80.

[9] C. Lemaitre, P. Hémon \& E. De Langre, 'Thin water film around a cable subject to wind', J. Wind Eng. Ind. Aerod., 95.9-11 (2007): 1,259-1,271. 
[10] C. Lemaitre, E. De Langre \& P. Hémon, 'Rainwater rivulet running on a stay cable subject to wind', Eur. J. Mech. B. Fluids, 29.4 (2010): 251-258.

[11] Y.Q. Ni, X.Y. Wang, Z.Q. Chen \& J.M. Ko, 'Field observations of rain-windinduced cable vibration in cable-stayed Dongting Lake Bridge', J. Wind Eng. Ind. Aerod., 95 (2007) 303-328.

[12] D. Zuo, N.P. Jones \& J.A. Main, 'Field observation of vortex-and rain-windinduced stay-cable vibrations in a three-dimensional environment', J. Wind Eng. Ind. Aerod., 96 (2008) 1,124-1,133.

[13] D. Zuo, N.P. Jones, 'Interpretation of field observations of wind-and rain-windinduced stay cable vibrations', J. Wind Eng. Ind. Aerod., 98 (2010) 73-87.

[14] H. Li, W.L. Chen, F. Xu, F.C. Li \& J.P. Ou, 'A numerical and experimental hybrid approach for the investigation of aerodynamic forces on stay cables suffering from rainwind induced vibration', J. Fluid Struct., 26.7-8 (2010): 1,195-1,215.

[15] P. Cheng, H. Li, D. Fuster, W.L Chen \& S. Zaleski, 'Multi-scale simulation of rainwater morphology evolution on a cylinder subjected to wind'. Comput. \& Fluids, 123 (2015): 112-121.

[16] O. Flamand, 'Rain-wind induced vibration of cables', J. Wind Eng. Ind. Aerod., 57 (1995) 353-362.

[17] M. Matsumoto, T. Yagi, S. Sakai, J. Ohya \& T. Okada, 'Steady wind force coefficients of inclined stay cables with water rivulet and their application to aerodynamics', Wind Struct., 8 (2005) 107-120.

[18] M. Gu, X.Q. Du \& S.Y. Li, 'Experimental and theoretical simulations on wind- 
rain-induced vibration of 3-D rigid stay cables', J. Sound Vib., 320 (2009) 184-200.

[19] M. Matsumoto, N. Shiraishi \& H. Shirato, 'Rain-wind induced vibration of cables of cable-stayed bridges', J. Wind Eng. Ind. Aerod., 43 (1992) 2011-2022.

[20] N. Cosentino, O. Flamand \& C. Ceccoli, 'Rain-wind induced vibration of inclined stay cables-Part I: Experimental investigation and physical explanation', Wind Struct., 6 (2003) 471-484.

[21] M. Gu \& X. Du, 'Experimental investigation of rain-wind-induced vibration of cables in cable-stayed bridges and its mitigation', J. Wind Eng. Ind. Aerod., 93 (2005) 79-95.

[22] F. Li, W. Chen, H. Li \& R. Zhang, 'An ultrasonic transmission thickness measurement system for study of water rivulets characteristics of stay cables suffering from wind-rain-induced vibration', Sens. Actuator A-Phys., 159 (2010) 12-23.

[23] X. Du, M. Gu \& S. Chen, 'Aerodynamic characteristics of an inclined and yawed circular cylinder with artificial rivulet', J. Fluid Struct., 43 (2013) 64-82.

[24] M. Matsumoto, T. Yagi, M. Goto \& S. Sakai, 'Rain-wind-induced vibration of inclined cables at limited high reduced wind velocity region', J. Wind Eng. Ind. Aerod., $91(2003) 1-12$.

[25] H. Jing, Y. Xia, H. Li, Y. Xu \& Y. Li, 'Study on the role of rivulet in rain-windinduced cable vibration through wind tunnel testing', J. Fluid Struct., 59 (2015) 316327.

[26] H. Jing, Y. Xia, H. Li, Y. Xu \& Y. Li, 'Excitation mechanism of rain-wind induced cable vibration in a wind tunnel', J. Fluid Struct., 68 (2017) 32-47. 
[27] E. de Langre, 'Frequency lock-in is caused by coupled-mode flutter', J. Fluid Struct., 22 (2006) 783-791.

[28] R. Violette, E. de Langre \& J. Szydlowski, 'A linear stability approach to vortexinduced vibrations and waves', J. Fluid Struct., 26 (2010) 442-466.

[29] M.L. Facchinetti, E. de Langre \& F. Biolley, 'Vortex-induced travelling waves along a cable', Eur. J. Mech. B. Fluids, 23 (2004) 199-208.

[30] H.M. Irvine, 'Cable Structures', The MIT Press, Cambridge, MA, (1981). 